\title{
Aplicabilidade da Classificaçáo Internacional de Funcionalidade, Incapacidade e Saúde (CIF) para a avaliaçáo de crianças com paralisia cerebral: uma revisão sistemática
}

\author{
Lílian de Fátima Dornelas ${ }^{a}$, Mariana Sivieri Lambertuccib, \\ Michelle de Lima Mello ${ }^{\mathrm{b}}$, Frederico Tadeu Deloroso ${ }^{\mathrm{b}}$ \\ aPrograma de Pós-Graduação em Ciências da Reabilitação, Escola de Educação Física, Fisioterapia e \\ Terapia Ocupacional, Universidade Federal de Minas Gerais - UFMG, Belo Horizonte, MG, Brasil \\ bUniversidade Federal de Uberlândia - UFU, Uberlândia, MG, Brasil
}

\begin{abstract}
Resumo: Objetivo: Examinar e sintetizar o conhecimento disponível na literatura sobre os instrumentos utilizados na perspectiva da funcionalidade em crianças com paralisia cerebral (PC). Método: A busca dos artigos foi realizada nas bases de dados eletrônicos Google Scholar, PubMed, Lilacs e Medline, publicados no período de janeiro de 2006 a dezembro de 2012, usando as palavras-chave: paralisia cerebral (cerebral palsy), criança (child), avaliação (assessment), combinada com Classificação Internacional da Funcionalidade - CIF (ICF). A busca limitou aos artigos originais escritos em português e inglês. Dez artigos foram selecionados para análise da revisão, de acordo com os critérios preestabelecidos. Resultados: Os autores propuseram instrumentos que poderiam padronizar a avaliação para classificação dos componentes "Estrutura e função do corpo", "Atividades e Participação" e "Fatores ambientais", como Gross Motor Function Measure (GMFM), o Pediatric Evaluation of Disability Inventory (PEDI), o Goal Attainment Scaling (GAS), o Manual Ability Classification System (MACS), o The Gross Motor Function Classification System (GMFCS), o Physicians Rating Scale (PRS), o Vineland Adaptive Behavior Scale (VABS), o Pediatric Functional Independence Measure (WeeFIM), o Gillette Functional Assessment Questionnaire (FAQ), o Pediatric Quality of Life Inventory (PedsQL), o Pediatric Outcomes Data Collection Instrument (PODCI), o Gillette Gait Index (GGI), o Energy Expenditure Index (EEI) e o Vécu et Santé Perçue de l'Adolescent (VSP-A). Conclusão: Os domínios "Estrutura e função do corpo" e "Atividades e Participação" são mais frequentemente classificados conforme a CIF em crianças com PC e possuem uma variedade de instrumentação para aplicabilidade da classificação.
\end{abstract}

Palavras-chave: Paralisia Cerebral, CIF, Avaliação, Criança.

\section{Applicability of the International Classification of Functioning, Disability and Health (ICF) for evaluation of children with cerebral palsy: a systematic review}

\begin{abstract}
Objective: To examine and synthesize the knowledge available in the literature on the instruments used in the perspective of functionality in children with cerebral palsy $(\mathrm{CP})$, and to review the literature evaluating the instruments used for the implementation of the International Classification of Functioning, Disability and Health (ICF) in children with CP. Method: The search was conducted in the electronic databases Google Scholar, PubMed, Lilacs and Medline, for articles published between January 2006 and December 2012, using the following keywords: cerebral palsy, child and assessment, combined with ICF. Ten articles were selected for analysis according to
\end{abstract}

Autor para correspondência: Lílian de Fátima Dornelas, Escola de Educação Física, Fisioterapia e Terapia Ocupacional, Universidade Federal de Minas Gerais, Avenida Presidente Antônio Carlos, 6627, Campus Pampulha, CEP 31270-901, Belo Horzionte, MG, Brasil, e-mail: liliandefatima@hotmail.com

Recebido em 19/7/2013; $1^{\text {a }}$ Revisão em 10/11/2013; Aceito em 11/12/2013. 
pre-established criteria. Results: The authors proposed tools that could standardize the assessment for classification of the components "Structure and function of the body", "Activities and Participation" and "Environmental Factors", proposing instruments such as Gross Motor Function Measure (GMFM), Pediatric Evaluation of Disability Inventory (PEDI), Goal Attainment Scaling (GAS), Manual Ability Classification System (MACS), Gross Motor Function Classification System (GMFCS), Physicians Rating Scale (PRS), Vineland Adaptive Behavior Scale (VABS), Pediatric Functional Independence Measure (Wee FIM), Gillette Functional Assessment Questionnaire (FAQ), Pediatric Quality of Life Inventory (PedsQL), Pediatric Outcomes Data Collection Instrument (PODCI), Gillette Gait Index (GGI), Energy Expenditure Index (EEI), and Vécu et Santé Perçue de l'Adolescent (VSP-A). Conclusion: The domains "Structure and function of the body" and "Activities and Participation" are often classified according to ICF in children with $\mathrm{CP}$, and they present a variety of instrumentation for applicability of classification.

Keywords: Cerebral Palsy, ICF, Assessment, Child.

\section{Introdução}

As avaliaçóes e tratamentos de crianças com paralisia cerebral (PC) dependem de modelos utilizados para conceitualizar doenças e desordens. A publicação, pela Organização Mundial da Saúde (OMS), da Classificação Internacional de Funcionalidade, Incapacidade e Saúde (CIF) sugere uma oportunidade para auxiliar a integração das várias perspectivas relativas à reabilitação de crianças com PC (ROTTA, 2002; ORGANIZAÇÃO..., 2002; BRASILEIRO; MOREIRA; JORGE, 2009a).

O impacto da CIF no tratamento e reabilitação de crianças com PC é bem descrito em dois aspectos: no pensamento clínico e na prática clínica. No pensamento clínico enfoca as deficiências que as crianças com PC possuem, colocando um valor igual na promoção funcional das atividades que elas realizam e na facilitação de sua participação total em todos os aspectos da vida. Outro fator a ser observado é que o ambiente no qual a criança está inserida contém a família e, consequentemente, devem ser propostos programas de suporte aos parentes, para que eles possam participar ativamente do tratamento e reabilitação da criança. Outro conceito que emerge do modelo da CIF envolve a consideração dos fatores que abrangem o que a criança consegue fazer no seu ambiente atual (desempenho) e a sua habilidade em executar uma tarefa ou ação (capacidade). Isto é, o envolvimento da criança em uma situaçáo de vida em seu contexto real e o seu nível máximo de funcionalidade de um domínio em dado momento (ORGANIZAÇÃO..., 2002; LEITE; PRADO, 2004; ALVES; SPALVIERI, 2007).

Com relação à prática clínica, o modelo da CIF serve de guia importante para a seleção de instrumentos de medição, os quais irão auxiliar no processo de decisão de metas a serem atingidas e na determinação de resultados significativos. Mas, para isso, o profissional deve estar familiarizado com os diversos instrumentos de avaliação existentes, para assim selecionar o mais adequado para o seu serviço ou pesquisa (ORGANIZAÇĀO..., 2002; FONSECA; LIMA, 2008). Pensando nisso, o objetivo do presente estudo é examinar e sintetizar o conhecimento disponível na literatura sobre os instrumentos utilizados na perspectiva da funcionalidade em crianças com PC.

\section{Método}

Os estudos foram selecionados por meio de busca eletrônica nas bases de dados Medline/ Pubmed; Medline/BVS; Lilacs/BVS; Google Scholar, as quais englobaram o maior número de artigos referente ao tema proposto, no presente estudo. A estratégia de busca nas bases de dados eletrônicas incluiu pesquisas publicadas entre janeiro de 2006 a dezembro de 2012. Os descritores de saúde usados para pesquisa foram paralisia cerebral (cerebral palsy), avaliação (assessment), criança (child) combinada com Classificação Internacional de Funcionalidade (International Classification of Functioning - ICF), e a busca se limitou aos artigos originais em português e inglês.

- Critérios de inclusão: estudos envolvendo crianças e/ou adolescentes com PC nas idades inferiores a 18 anos com aplicação de instrumentos para avaliação e ou intervenção com fins fisioterapêuticos.

- Critérios de exclusão: estudos de caso, séries de casos, revisão da literatura, artigos com informaçôes duplicadas ou que somente citaram os instrumentos ou, ainda, que não utilizaram para avaliação em crianças com PC e sobre validação de instrumentos.

Os autores utilizaram o modelo PICO (AKOBENG, 2005) para descrever todos os componentes relacionados ao problema identificado 
e estruturar a pergunta da pesquisa, que foi: Quais os instrumentos utilizados para avaliar crianças e/ ou adolescentes nas idades inferiores a 18 anos com PC? (Tabela 1).

Uma vez que a questão de pesquisa foi formulada, a etapa seguinte foi o início da busca bibliográfica de evidências, que foi esquematizada nas seguintes etapas apresentadas a seguir: a) Seleção dos termos de busca: palavras-chave delimitadas no método com a utilização dos operadores booleanos AND e OR.

Feitas as buscas, inicialmente os títulos dos trabalhos localizados foram analisados, descartando aqueles não pertinentes. Na sequência, foram lidos os resumos, sendo mantidos os artigos específicos sobre o tema proposto. A sessão de metodologia desses artigos foi lida, sendo aplicados os critérios de inclusão, até se chegar aos trabalhos finais, específicos sobre o tema em estudo. Esses trabalhos foram lidos na íntegra, com análise detalhada da metodologia e dos resultados, de forma a construir a presente revisão. Assim, foram incluídos para análise crítica na presente revisão dez artigos, como ilustrado na Figura 1.

Para a inclusão de artigos de boa qualidade metodológica foi feita uma avaliação dos estudos experimentais, por meio da escala PEDro e dos estudos observacionais, com base nas recomendaçôes STROBE (Strengthening The Reporting of Observational Studies in Epidemiology), por um único revisor independente. A avaliação A escala PEDro é baseada na lista Delphi e foi criada pela Physiotherapy Evidence Database. É constituída de 11 itens em que cada critério vale um ponto. Estudos com pontuação menor que três são considerados de baixa qualidade metodológica (MAHER et al., 2003). A lista de verificação STROBE foi recentemente traduzida e adaptada para o português. Contém 22 itens, porém ela não foi desenvolvida com o objetivo de avaliar a qualidade metodológica de estudos, contudo vem sendo comumente usada no Brasil para esse fim. Pesquisadores brasileiros estabeleceram três categorias para classificar a qualidade dos artigos: $A=$ caso o estudo preencha $80 \%$ ou mais dos critérios estabelecidos no STROBE; B = caso preencha de $50 \%$ a $79 \%$ dos critérios STROBE e C = quando menos de $50 \%$ dos critérios forem preenchidos (MALTA et al., 2010).

Todos os artigos atingiram pontuação correspondente a $80 \%$ nos itens estabelecidos pelas referidas escalas, apresentando boa qualidade metodológica.

\section{Resultados}

Para facilitar a apreciação das informaçóes obtidas nos diferentes estudos, os dados foram resumidos e apresentados na Tabela 2, no qual foram descritas informaçóes dos artigos segundo autor, participantes, objetivo, intervenção, instrumentação e resultado encontrado.

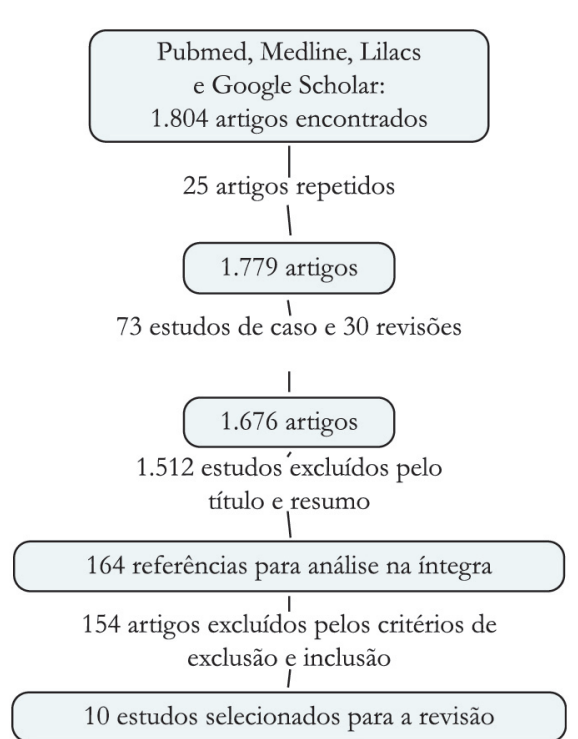

Figura 1. Etapas da revisão.

Tabela 1. Descrição dos componentes do PICO* para a presente revisão sistemática.

\begin{tabular}{ccl}
\hline Acrônimo & Definição & \multicolumn{1}{c}{ Descrição } \\
\hline P & Paciente & $\begin{array}{l}\text { Crianças e/ou adolescentes nas idades inferiores a 18 anos com diagnóstico } \\
\text { de paralisia cerebral, incluindo os tipos: hemiplegia, diplegia e tetra ou } \\
\text { quadriplegia. }\end{array}$ \\
\hline I & Intervenção & $\begin{array}{l}\text { Instrumentos de medição nacionais e/ou internacionais utilizados para avaliar } \\
\text { a criança e/ou adolescente com paralisia cerebral. }\end{array}$ \\
\hline C & Comparação & $\begin{array}{l}\text { Comparação com outras crianças utilizando os mesmos testes ou nenhuma } \\
\text { comparação. }\end{array}$ \\
\hline O & Observação & $\begin{array}{l}\text { Resultado do teste após a avaliação ou intervenção ou programa de tratamento } \\
\text { fisioterapêutico. }\end{array}$ \\
\hline
\end{tabular}

*Akobeng (2005). 


\begin{tabular}{|c|c|c|c|}
\hline & 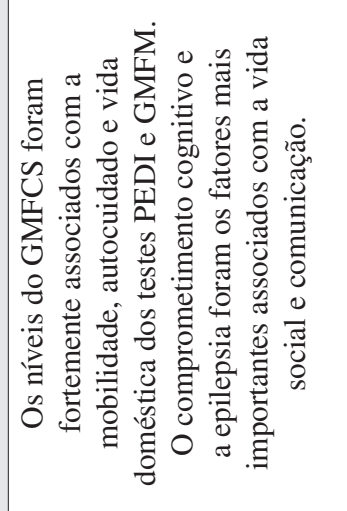 & 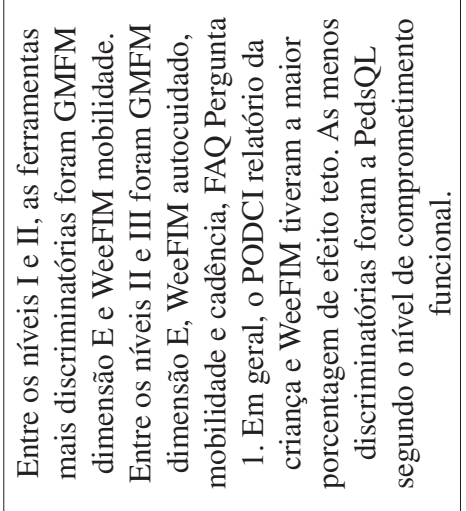 & 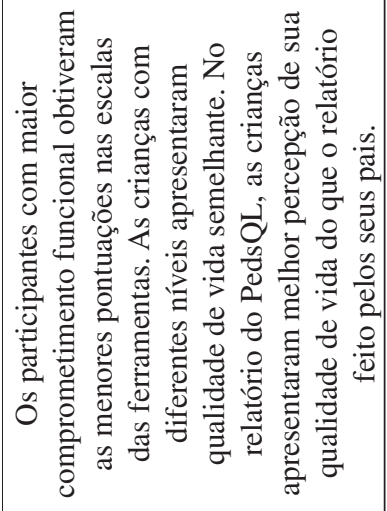 \\
\hline | & 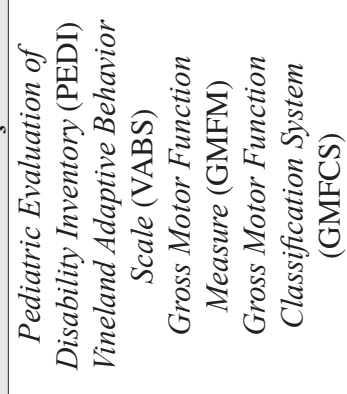 & 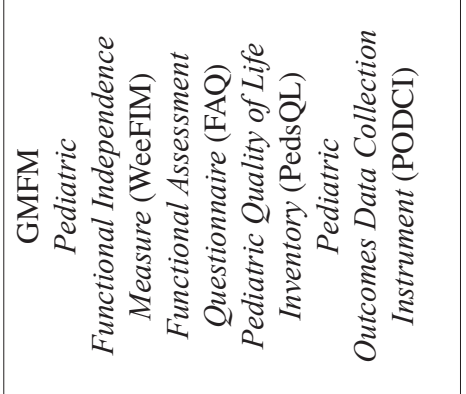 & 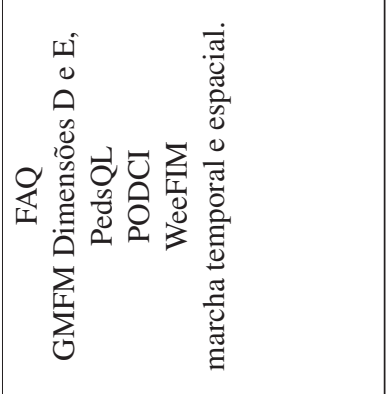 \\
\hline 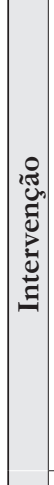 & 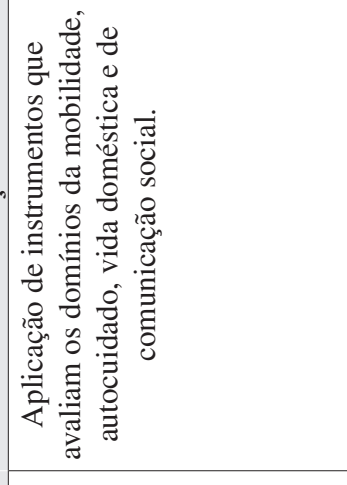 & 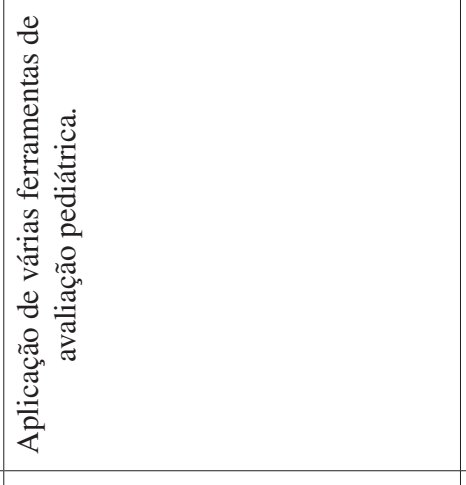 & 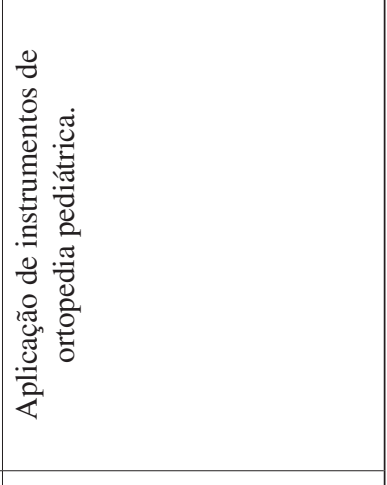 \\
\hline 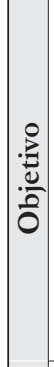 & 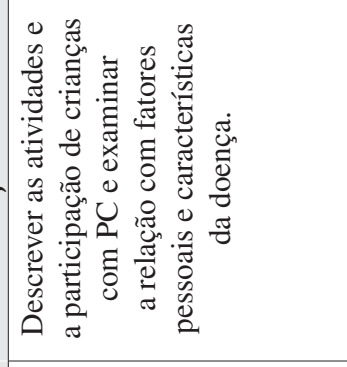 & 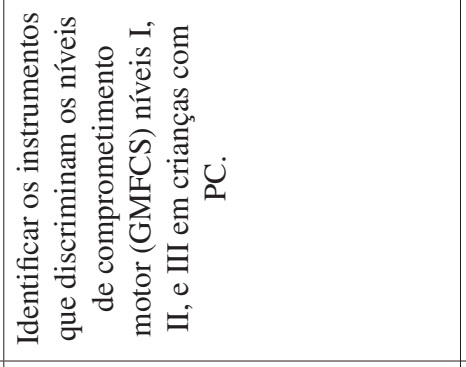 & 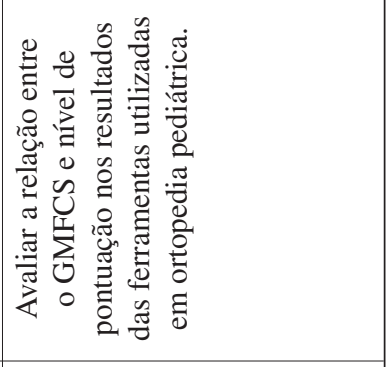 \\
\hline | & 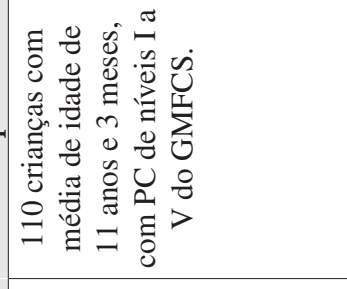 & 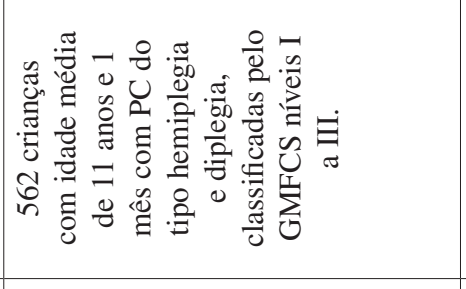 & 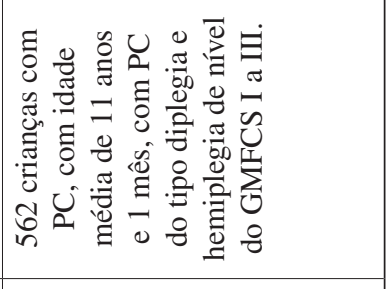 \\
\hline 节 & 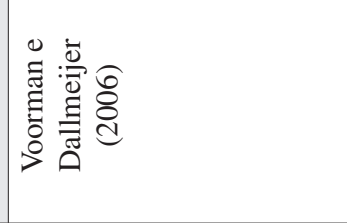 & 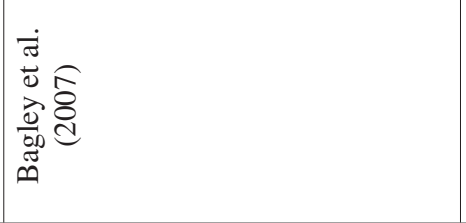 & 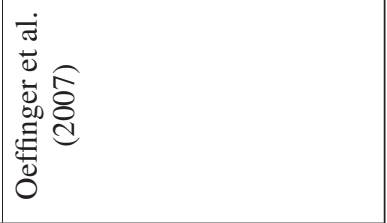 \\
\hline
\end{tabular}




\begin{tabular}{|c|c|c|c|c|}
\hline & 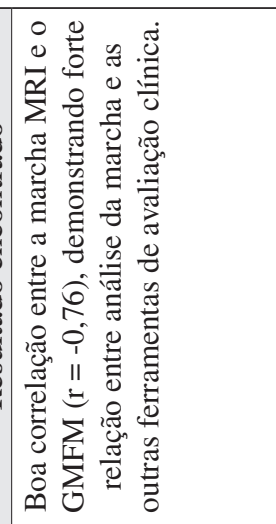 & 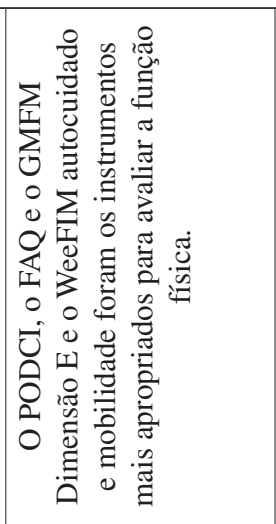 & 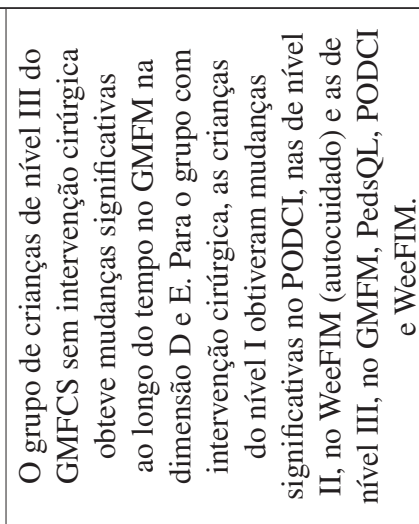 & 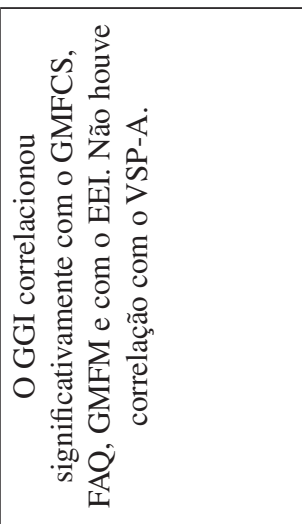 \\
\hline 赵 & 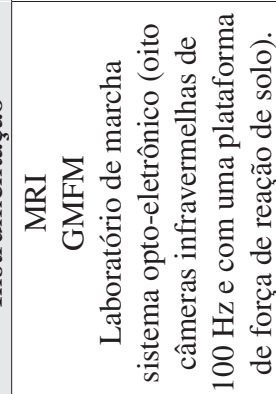 & 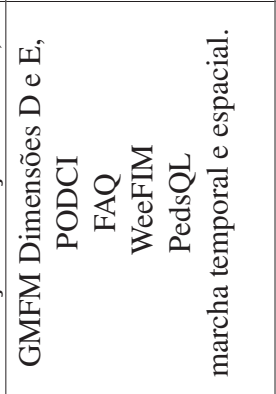 & 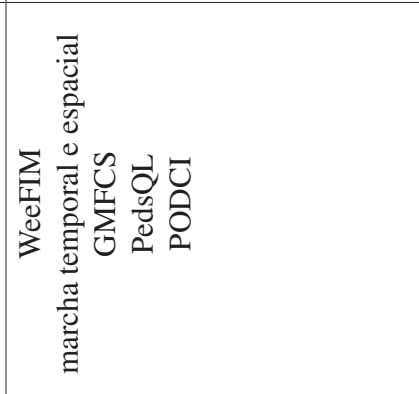 & 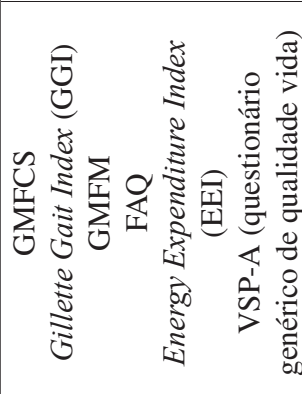 \\
\hline & 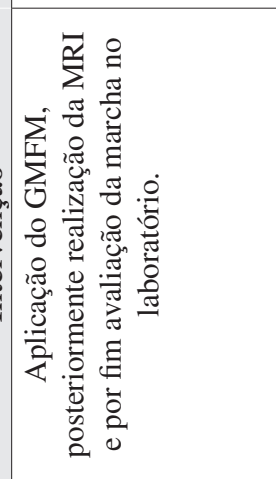 & 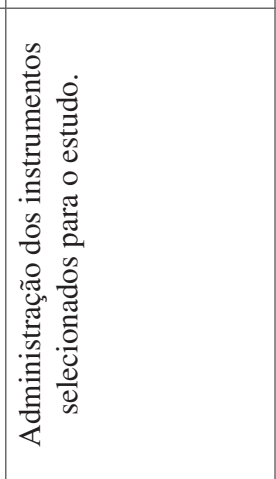 & 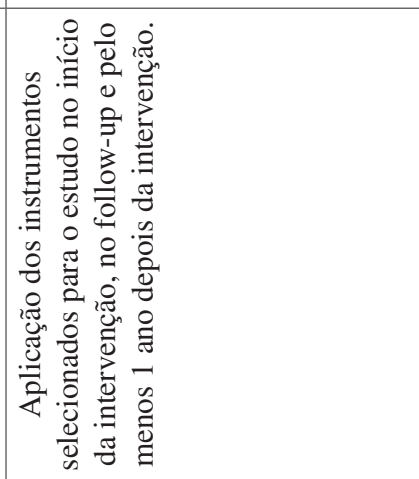 & 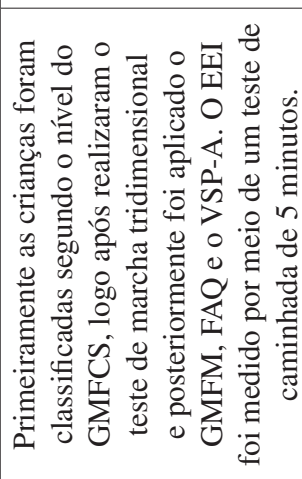 \\
\hline & 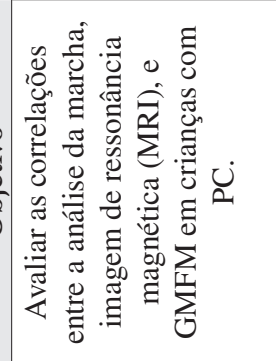 & 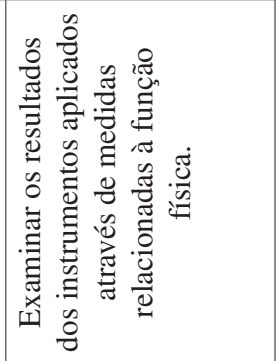 & 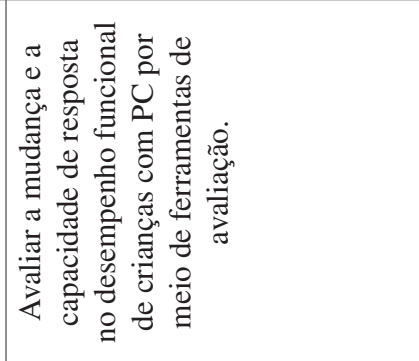 & 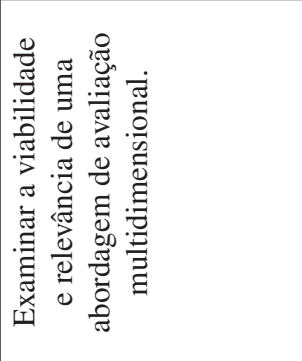 \\
\hline 音 & 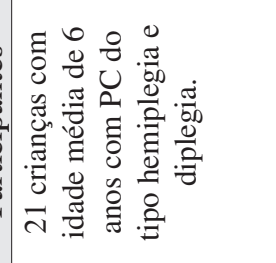 & 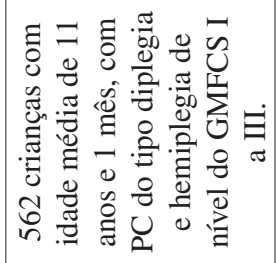 & 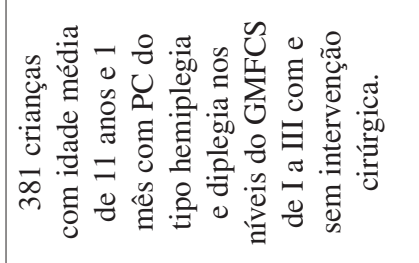 & 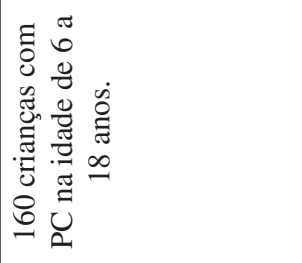 \\
\hline & 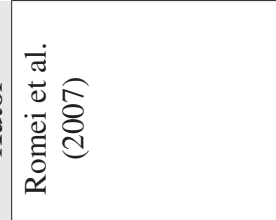 & 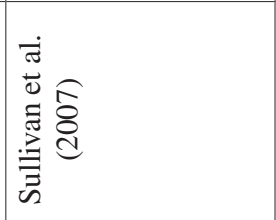 & 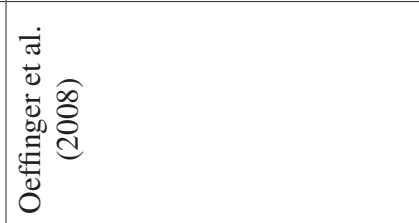 & 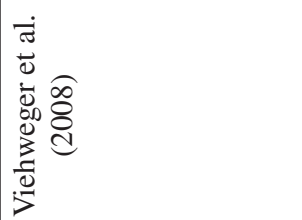 \\
\hline
\end{tabular}




\begin{tabular}{|c|c|c|c|}
\hline & 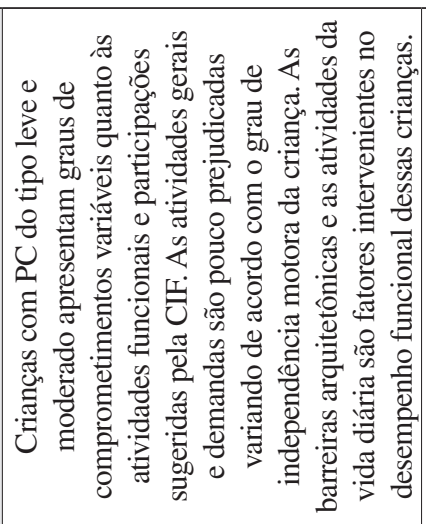 & 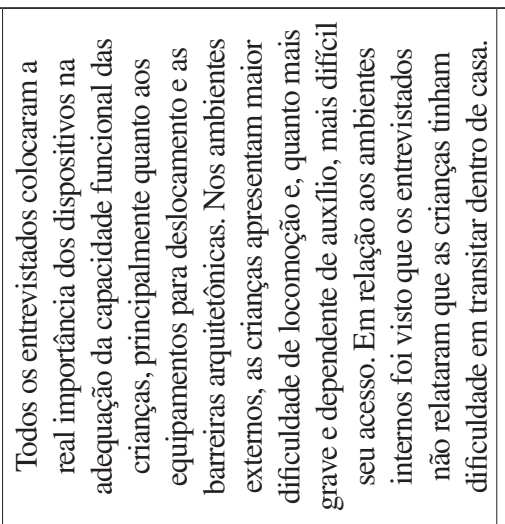 & 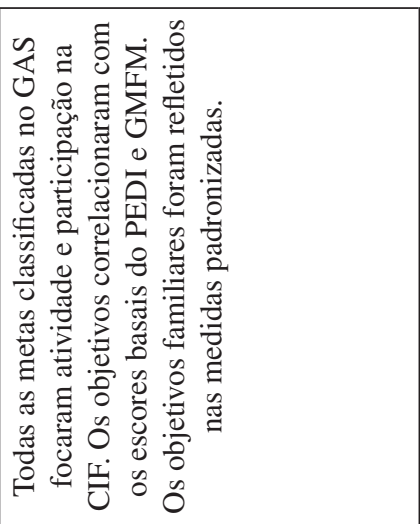 \\
\hline & 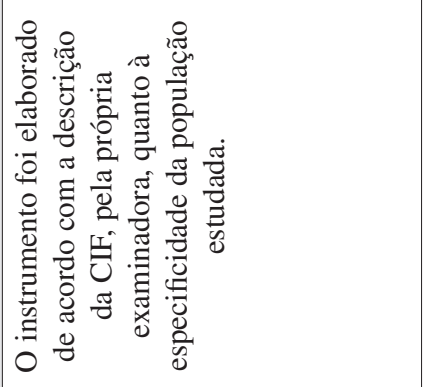 & 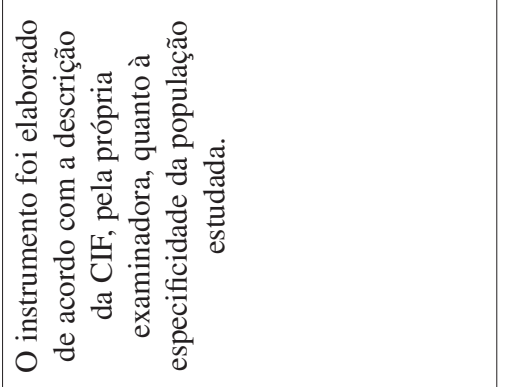 & 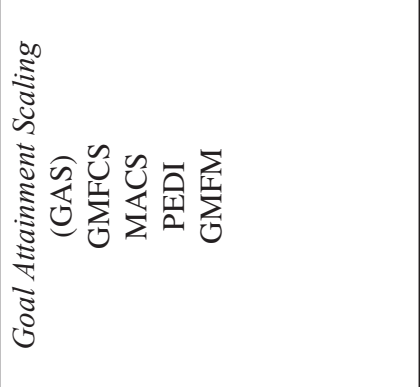 \\
\hline & 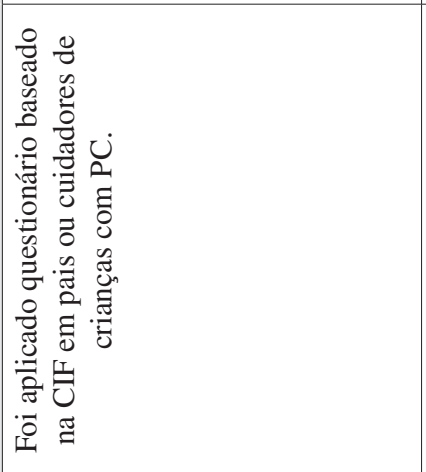 & 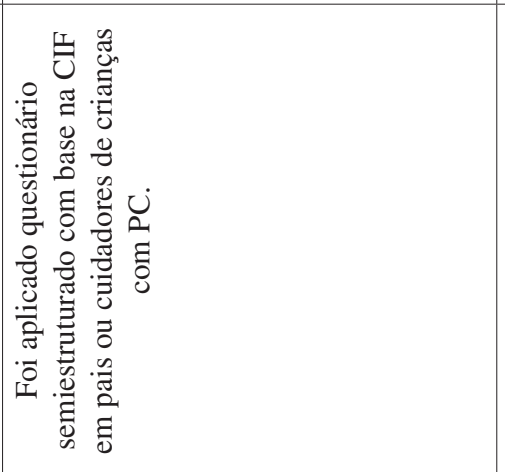 & 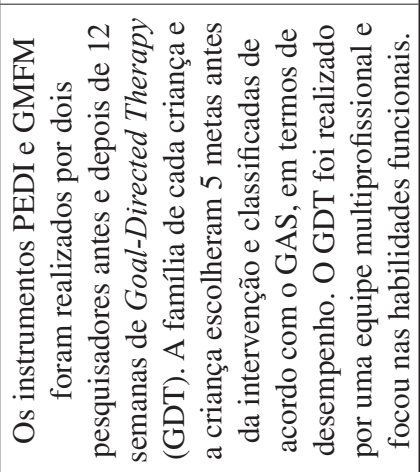 \\
\hline (0) & 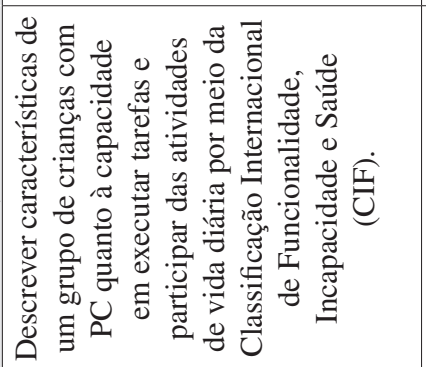 & 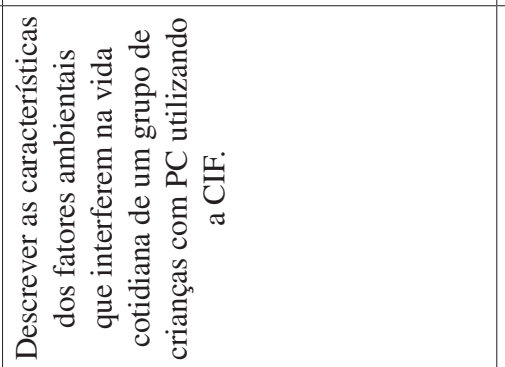 & 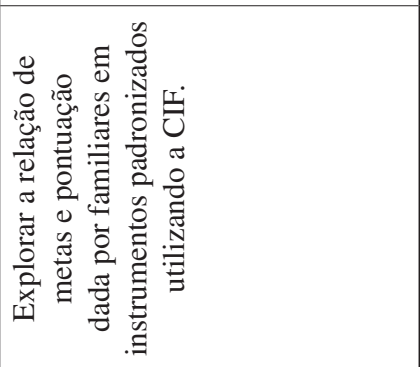 \\
\hline 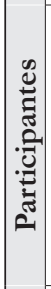 & 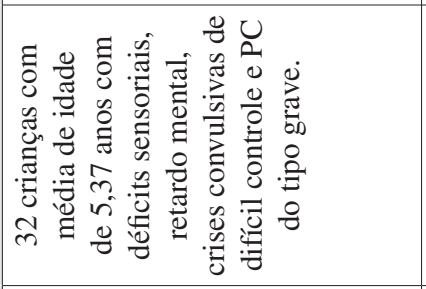 & 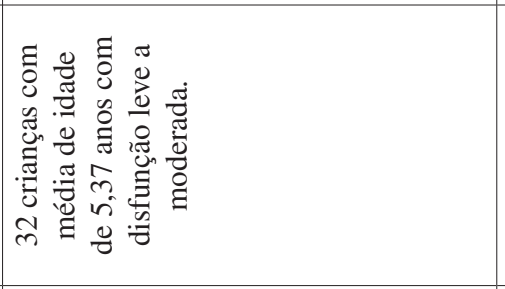 & 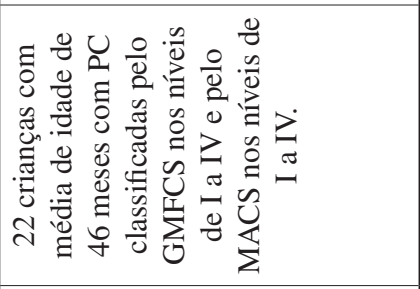 \\
\hline & 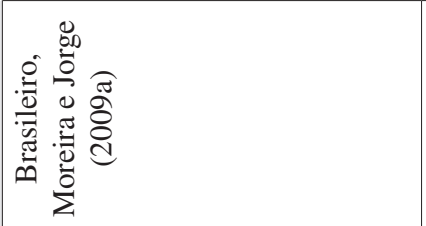 & 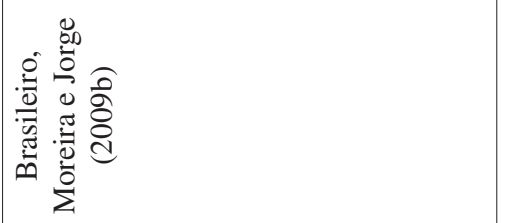 & 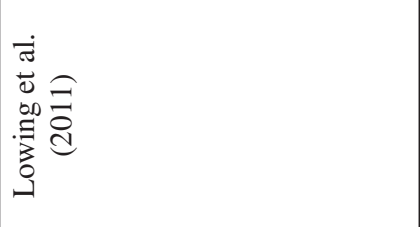 \\
\hline
\end{tabular}


Dentre os estudos selecionados, os autores propuseram instrumentos que poderiam padronizar a avaliação para classificação dos componentes "Estrutura e função do corpo", "Atividades e Participação" e "Fatores ambientais", propondo instrumentos como o Gross Motor Function Measure (GMFM), o Pediatric Evaluation of Disability Inventory (PEDI), o Goal Attainment Scaling (GAS), o Manual Ability Classification System (MACS), o The Gross Motor Function Classification System (GMFCS), Physicians Rating Scale (PRS), o Vineland Adaptive Behavior Scale (VABS), Pediatric Functional Independence Measure (WeeFIM), Gillette Functional Assessment Questionnaire (FAQ), o Pediatric Quality of Life Inventory (PedsQL), o Pediatric Outcomes Data Collection Instrument (PODCI), o Gillette Gait Index (GGI), o Energy Expenditure Index (EEI) e o Vécu et Santé Perçue de l'Adolescent (VSP-A). Informaçóes detalhadas dos instrumentos segundo a aplicabilidade da CIF encontram-se na Tabela 3.

\section{Discussão}

Todos os instrumentos identificados neste estudo foram utilizados para avaliar crianças com PC. Cada instrumento possui objetivo específico, sendo alguns adequados para avaliar função, outros para diagnóstico de lesóes neurológicas e outros ainda para avaliar o desempenho. Entretanto, é necessária a escolha correta do instrumento, pois cada um tem uma finalidade específica, de acordo com os objetivos definidos para coleta de dados e implementação da detecçáo ou intervenção a ser realizada (PINTO; VILANOVA; VIEIRA, 1997).

É de grande importância clínica que seja utilizado um instrumento com normas e critérios de referência, para mensuração confiável de dados clínicos relevantes. Observa-se, com base nos estudos analisados, o pequeno número de instrumentos com validação e padronização para a população brasileira, sendo essencial investir na padronização dos instrumentos existentes em nosso país ou publicados no exterior, para permitir a avaliação confiável em crianças com PC. Os instrumentos de avaliação para crianças com PC devem ser capazes de mensurar o desenvolvimento, suas principais funções, além de acompanhar a evoluçáo durante todo o tratamento, permitindo assim uma linguagem única entre a equipe multidisciplinar (PINTO; VILANOVA; VIEIRA, 1997).

Dos instrumentos encontrados no presente estudo, o The Gross Motor Function Classification System (GMFCS), o Manual Ability Classification System (MACS) e a Classificação Internacional de
Funcionalidade (CIF) são sistemas de classificação, sendo o GMFCS e MACS específicos para PC. Destaca-se o GMFCS pela frequência de seu uso nos estudos da presente revisão. É um sistema baseado no movimento autoiniciado com ênfase no sentar, transferências e mobilidade. Possui cinco níveis que incluem a faixa etária entre antes do $2^{\circ}$ ano de vida até os 18 anos, sendo que dos 12 aos 18 anos de idade enfatiza os conceitos inerentes à CIF. A finalidade desse sistema é determinar qual o nível que melhor representa as atuais competências e limitaçóes na função motora global (PALISANO; ROSEMBAUM; RUSSELL, 1997). Na presente revisão, observou-se que, com o uso dessa classificação, permitiu-se padronizar o nível de comprometimento em relação à função motora grossa.

Já o MACS classifica como as crianças com PC usam suas mãos para manipular objetos em atividades diárias em cinco níveis que incluem a faixa etária de 4 a 18 anos. Os níveis são baseados na habilidade da criança em iniciar sozinha a manipulação de objetos e a necessidade de assistência ou adaptação para realizar atividades manuais na vida diária. É uma descrição funcional que pode ser usada como complemento do diagnóstico de PC e seus subtipos (ELIASSON et al., 2006).

A CIF descreve aspectos da funcionalidade e da incapacidade e fatores contextuais relacionados às condiçôes de saúde do indivíduo. A versão em língua portuguesa foi traduzida com o título de Classificação Internacional de Funcionalidade, Incapacidade e Saúde. A CIF é um modelo de Classificação da Organizaçâo Mundial da Saúde (OMS) baseado na etiologia, anatomia e causas das lesões, porém não responde a questôes relacionadas às consequências das doenças ou ao impacto da incapacidade ao longo dos anos na vida do indivíduo. Ela organiza a informação em duas partes, a primeira em funcionalidade e incapacidade e a segunda em fatores contextuais. Cada parte é subdividida em componentes, sendo a primeira em corpo (estrutura e função), atividades e participação e a segunda parte em fatores ambientais e pessoais (ORGANIZAÇÃO..., 2002). Atualmente, a CIF tem se tornado modelo de avaliação em várias patologias e, pelo que se nota na presente revisão, ela tem contribuído para uma avaliação mais completa em crianças com PC.

Além dos instrumentos de classificaçáo foram encontrados, na revisão, os instrumentos Gross Motor Function Measure (GMFM), Pediatric Evaluation of Disability Inventory (PEDI), Goal Attainment Scaling (GAS), Physicians Rating Scale (PRS), Vineland Adaptive Behavior Scale (VABS), Pediatric Functional Independence Measure (WeeFIM), Gillette Functional Assessment Questionnaire (FAQ), Pediatric Quality 


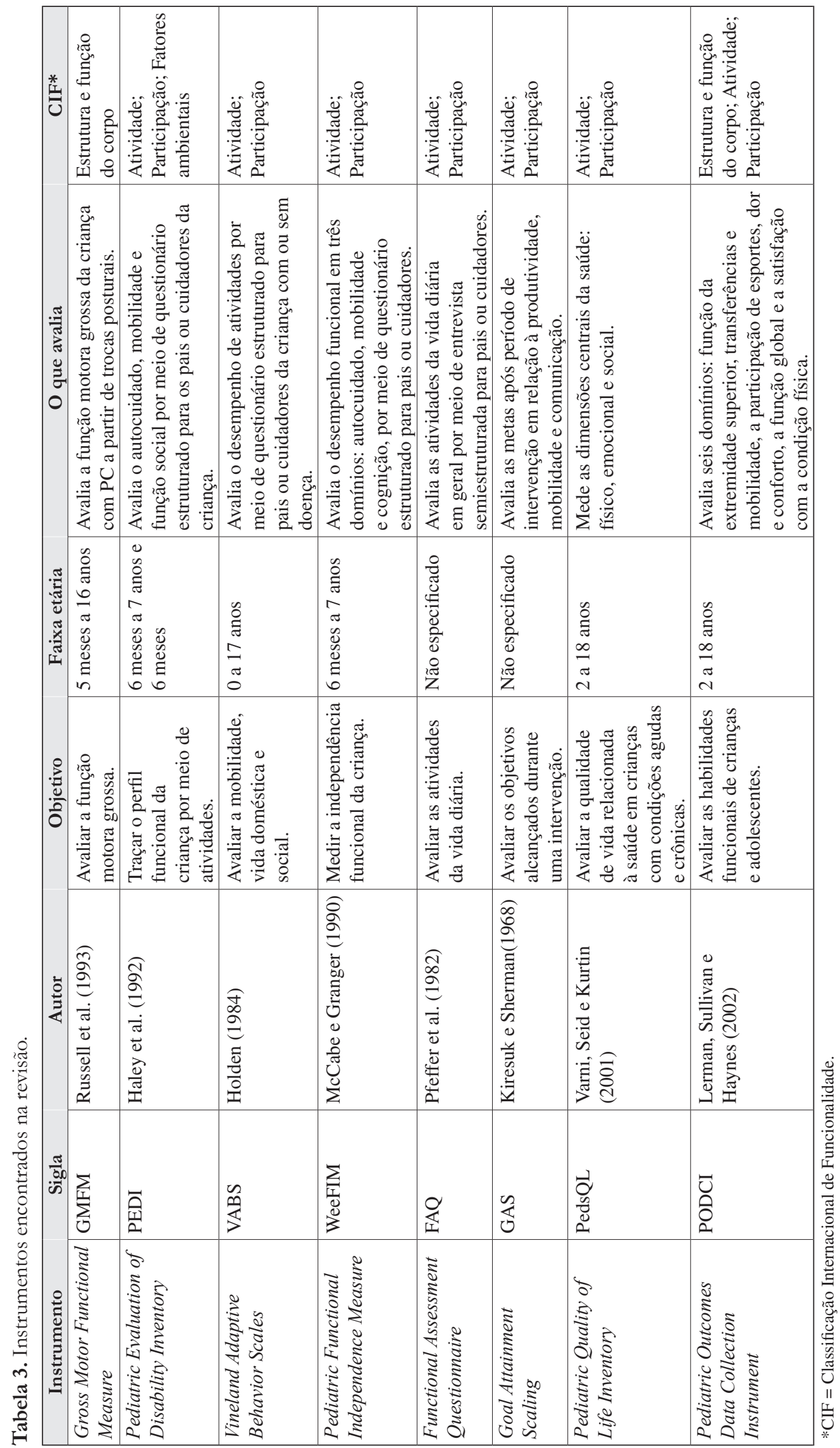




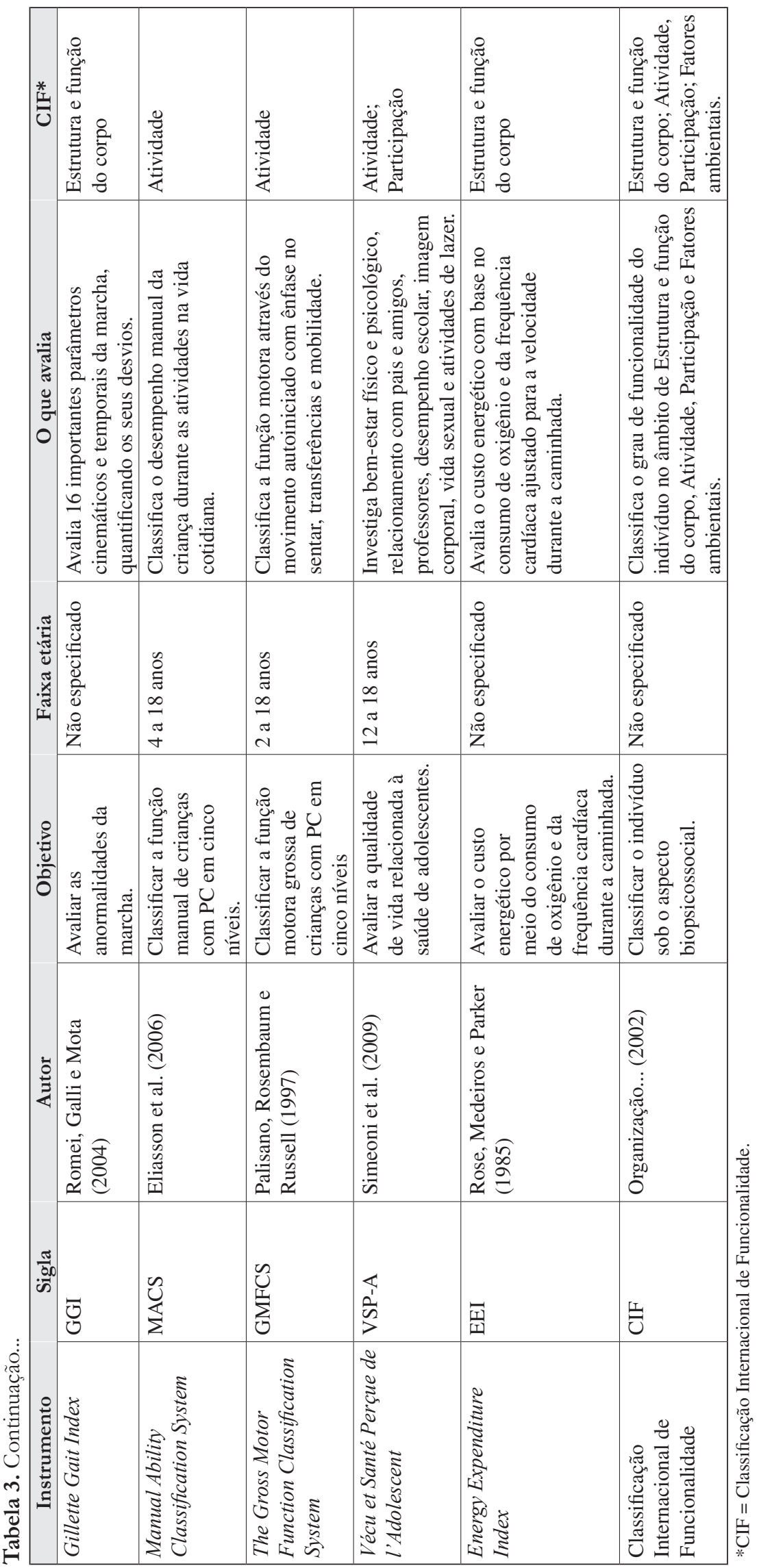


of Life Inventory (PedsQL), Pediatric Outcomes Data Collection Instrument (PODCI), Gillette Gait Index (GGI), Energy Expenditure Index (EEI) e Vécu et Santé Perçue de l'Adolescent (VSP-A). São testes que avaliam o paciente e permitem que sejam usados antes e depois de uma intervenção terapêutica, sendo o GMFM específico para PC e os outros podem abranger diferentes patologias.

Destaca-se, pela frequência de uso, o GMFM, que é um instrumento que descreve o nível de função motora grossa, mensurando e documentando mudanças motoras. Tal avaliação é agrupada em cinco dimensôes da função motora grossa, e o tempo de aplicação varia em torno de 45 minutos, é de baixo custo e fácil aplicaçấo. Tal teste pode ser usado tanto na prática clínica quanto em pesquisas científicas (RUSSELL, 1993). Na revisão foi visto que, apesar desse teste focar somente a função motora grossa, ou seja, a categoria "Estrutura e função do corpo" pela CIF, é um instrumento que atende o objetivo de avaliação desse domínio e é bastante utilizado na prática clínica em diversas situações em crianças com PC. Além disso, o GMFM quando aplicado em associação com outros testes corrobora para uma avaliação mais funcional (FONSECA; LIMA, 2008; ROTTA, 2002).

Vale ressaltar o Pediatric Evaluation of Disability Inventory - PEDI (HALEY et al., 1992), que é um inventário funcional norte-americano e foi traduzido para o português (MANCINI, 2005) e adaptado para contemplar as especificidades socioculturais do Brasil. O PEDI é realizado por meio de entrevista com pais ou responsáveis, que informam sobre o desempenho típico da criança em casa. Os itens do teste avaliam aspectos funcionais do desenvolvimento de crianças com idade entre seis meses e sete anos e seis meses, em três áreas de desempenho: autocuidado, mobilidade e função social. O PEDI pode ser aplicado em 45 a 60 minutos e apresenta estudos que dão suporte à confiabilidade e à validade.

Contudo, observa-se na presente revisão que, para uma avaliação global e melhor mensuração da funcionalidade da criança com PC, é mais eficaz a associaçáo de instrumentos na avaliaçáo e intervenção, pois dessa maneira tem-se evidenciada maior sensibilidade para detectar alteraçôes motoras, como também o fornecimento de dados para o direcionamento de metas e planejamento para intervençôes terapêuticas. Lembrando que a avaliação dessas crianças compreende desde a coleta de dados clínicos e subjetivos à aplicação de testes, e estes últimos devem ser usados de maneira criteriosa.

\section{Conclusão}

É primordial fazer o uso de instrumentação correta, com normas e/ou critérios de referência adequados, para avaliar, diagnosticar e planejar o tratamento de crianças com PC. Os domínios "Estrutura e função do corpo", "Atividade e Participaçáo" são frequentemente classificados conforme a CIF em crianças com PC e possuem uma variedade de instrumentação para aplicabilidade da classificação.

Na prática clínica, a CIF pode ser usada para guiar o processo de avaliação, planejamento da intervenção e avaliação do tratamento realizado em crianças com PC, onde diferentes instrumentos podem ser utilizados para cobrir os vários conceitos do modelo. No contexto da avaliação, para identificar o nível de funcionalidade, no planejamento da intervençáo, para auxiliar na escolha de tratamentos que maximizem a funcionalidade e na avaliação do tratamento, para caracterizar os resultados da intervenção e sua utilidade (ORGANIZAÇÃO..., 2002).

\section{Referências}

ALVES, F. D.; SPALVIERI, D. F. Fisioterapia aquática aplicada à pediatria. In: SACCHELLE, T.; ACCACIO, L. M. P.; RADL, A. L. M. Fisioterapia aquática. São Paulo: Manole, 2007. p. 588-598.

AKOBENG, A. K. Principles of evidence based medicine. Archives Disease in Chilhood, London, v. 90, n. 8, p. 837-840, 2005. PMid:16040884 PMCid:PMC1720507. http://dx.doi.org/10.1136/adc.2005.071761

BAGLEY, A. M. et al. Outcome assessments in children with cerebral palsy. Part II: discriminatory ability of outcome tools. Developmental Medicine and Child Neurology, Oxford, v. 49, n. 3, p. 181-186, 2007. PMid:17355473. http://dx.doi.org/10.1111/j.1469-8749.2007.00181.x

BRASILEIRO, I. C.; MOREIRA, T. M. M.; JORGE, M. S. B. Atividade e participação de crianças com paralisia cerebral conforme a Classificação Internacional de Funcionalidade, Incapacidade e Saúde. Revista Brasileira de Enfermagem, São Paulo, v. 62, n. 4, p. 503-511, 2009a. http://dx.doi.org/10.1590/S0034-71672009000400002

BRASILEIRO, I. C.; MOREIRA, T. M. M.; JORGE, M. S. B. Interveniência dos fatores ambientais na vida de crianças com Paralisia Cerebral. Acta Fisiatrica, São Paulo, v. 16, n. 3, p. 132-137, 2009b.

ELIASSON, A. C. et al. O Sistema de Classificação de Habilidade Manual (MACS) para crianças com paralisia cerebral: desenvolvimento da escala e evidência de validade e confiabilidade. Developmental Medicine and Child Neurology, Oxford, v. 48, n. 7, p. 549-554, 2006. PMid:16780622. http://dx.doi. org/10.1017/S0012162206001162 
FONSECA, L. F.; LIMA, C. L. Paralisia cerebral: neurologia, ortopedia e reabilitação. Rio de Janeiro: Medbook, 2008.

HALEY, S. M. et al. Pediatric evaluation of disability inventory (PEDI): development, standardization and administration manual (version 1.0). Boston: PEDI Research Group, New England Medical Center Inc., 1992.

HOLDEN, R. H. Vineland adaptive behavior scales. Test Critiques, Kansas, v. 1, p. 715-719, 1984.

KIRESUK, T.; SHERMAN R. Goal attainment scaling: a general method for evaluating comprehensive community health programs. Community Mental Health Journal, New York, v. 4, n. 6, p. 443-453, 1968. PMid:24185570. http://dx.doi.org/10.1007/BF01530764

LEITE, J. M. R.; PRADO, G. F. Paralisia cerebral: aspectos fisioterapêuticos e clínicos. Revista Neurociências, São Paulo, v. 12, n. 41, p. 41-45, 2004. http://dx.doi. org/10.4181/RNC.2004.12.41

LERMAN, J. A.; SULLIVAN, E.; HAYNES, R. J. The Pediatric Outcomes Data Collection Instrument (PODCI) and functional assessment in patients with adolescent or juvenile idiopathic scoliosis and congenital scoliosis or kyphosis. Spine, Philadelphia, v. 27 , n. 18 , p. 2052-2057, 2002. http://dx.doi. org/10.1097/00007632-200209150-00016

LOWING, K. et al. Exploring the relationship of family goals and scores on standardized measures in children with cerebral palsy using the ICF-CY. Developmental Neurorehabilitation, London, v. 14, n. 2, p. 79-86, 2011. PMid:21410399. http://dx.doi.org/10.3109/17518423.2 011.552088

MAHER, C. G. et al. Reliability of the PEDro scale for rating quality of randomized controlled trials. Physical Therapy, Alexandria, v. 83, n. 8, p. 713-721, 2003.

MALTA, M. et al. Iniciativa STROBE: subsídios para a comunicação de estudos observacionais. Revista de Saúde Pública, São Paulo, v. 44, n. 3, p. 559-565, 2010. http://dx.doi.org/10.1590/S0034-89102010000300021

MANCINI, M. C. Inventário de Avaliação Pediátrica de Incapacidade (PEDI): manual da versáo brasileira adaptada. Belo Horizonte: UFMG, 2005. (Coleção Didática).

MCCABE, M.; GRANGER, C. Content validity of a pediatric functional independence measure. Applied Nursing Research, Philadelphia, v. 3, n. 3, p. 120-122, 1990. http://dx.doi.org/10.1016/S0897-1897(05)80128-4

OEFFINGER, D. et al. Outcome assessments in children with cerebral palsy, Part I: descriptive characteristics of GMFCS Levels I to III. Developmental Medicine and, Child Neurology, Oxford, v. 49, n. 3, p. 172-180, 2007.

OEFFINGER, D. et al. Outcome tools used for ambulatory children with cerebral palsy: responsiveness and minimum clinically important differences. Developmental Medicine and Child Neurology, Oxford, v. 50, n. 12, p. 918-925, 2008. PMid:19046185 PMCid:PMC2990955. http://dx.doi.org/10.1111/j.1469-8749.2008.03150.x

ORGANIZAÇÃO MUNDIAL DE SAÚDE - OMS. A Classificaçāo Internacional de Funcionalidade, Incapacidade e Saúde. Genebra, 2002.
PALISANO, R.; ROSEMBAUM, P. L.; RUSSELL, D. Development and reliability of a system to classify gross motor function in children with cerebral palsy. Developmental Medicine and Child Neurology, Oxford, v. 39, n. 4, p. 214-223, 1997. http://dx.doi. org/10.1111/j.1469-8749.1997.tb07414.x

PFEFFER, R. I. et al. Measurement of functional activities in older adults in the community. Journal Gerontology, Washington, v. 1, n. 3, p. 323-329, 1982. http://dx.doi. org/10.1093/geronj/37.3.323

PINTO, E. B.; VILANOVA, L. C. P.; VIEIRA, R. M. $O$ desenvolvimento do comportamento da criança no primeiro ano de vida. São Paulo: Casa do Psicólogo: FAPESP, 1997.

ROMEI, M. et al. Analysis of the correlation between three methods used in the assessment of children with cerebral palsy. Functional Neurology, Roma, v. 22, n. 1, p. 17-21, 2007.

ROMEI, M.; GALLI, M.; MOTTA, F. Use of the normalcy index for the evaluation of gait pathology. Gait Posture, Oxford, v. 19, n. 1, p. 85-90, 2004. http:// dx.doi.org/10.1016/S0966-6362(03)00017-1

ROSE, J.; MEDEIROS, J. M.; PARKER, R. Energy cost index as an estimate of energy expenditure of cerebral-palsied children during assisted ambulation. Developmental Medicine Child Neurology, Oxford, v. 27, n. 4 , p. $485-490,1985$. http://dx.doi. org/10.1111/j.1469-8749.1985.tb04572.x

ROTTA, N. T. Paralisia cerebral, novas perspectivas terapêuticas. Jornal de Pediatria, Rio de Janeiro, v. 78, p. S48-S54, 2002. Suplemento 1.

RUSSELL, P. et al. Gross Motor Function Measure (GMFM-66 and GMFM-88). User's manual. London: Mac Keith Press, 1993.

SIMEONI, M. C. et al. Validation of a French healthrelated quality of life instrument for adolescents: the VSP-A. Quality of Life Research, Oxford, v. 9, n. 4, p. 393-403, 2009. http://dx.doi.org/10.1023/A:1008957104322

SULLIVAN, E. et al. Relationships among functional outcome measures used for assessing children with ambulatory CP. Developmental Medicine and Child Neurology, Oxford, v. 49, n. 5, p. 338-344, 2007. PMid:17489806. http://dx.doi. org/10.1111/j.1469-8749.2007.00338.x

VARNI, J. W.; SEID, M.; KURTIN, P. S. The PedsQL 4.0: reliability and validity of the Pediatric Quality of Life Inventory generic core scales in healthy and patient populations. Medical Care, Philadelphia, v. 39, n. 8 , p. 800-812, 2001. http://dx.doi. org/10.1097/00005650-200108000-00006

VIEHWEGER, E. et al. Multidimensional outcome assessment in cerebral palsy is it feasible and relevant? Journal Pediatric Orthopaedics, Philadelphia, v. 28, n. 5, p. 576-583, 2008.

VOORMAN, J. M.; DALLMEIJER, A. J. Activities and participation of 9- to 13-year-old children with cerebral palsy. Clinical Rehabilitation, London, v. 20, n. 11, p. 937-948, 2006. PMid:17065537. http://dx.doi. org/10.1177/0269215506069673 


\section{Contribuição dos Autores}

Lílian de Fátima Dornelas: concepção do texto, organização de fontes e revisão final. Mariana Sivieri Lambertucci: concepção do texto manuscrito e organização de fontes. Michelle de Lima Mello: organização de fontes. Frederico Tadeu Deloroso: orientaçáo e revisão final. Todos os autores aprovaram a versão final do texto. 